\title{
AVALIAÇÃO DA QUALIDADE DA ÁGUA DO ABASTECIMENTO PÚBLICO DO MUNICÍPIO DE JUAZEIRO DO NORTE, CE
}

Evaluation of the water quality of the public supply of the municipality of Juazeiro do Norte, $C E$

Evaluación de lacalidad de la agua del suministro público del municipio de Juazeiro do Norte, $C E$

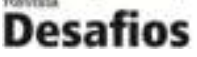

Artigo Original

Original Article

Artículo Original

\section{William Santana Alves ${ }^{1}$, Toshik Iarley da Silva ${ }^{* 2}$, Deivid Antônius da Silva Marrom ${ }^{3}$,} Tainá Macêdo dos Santos ${ }^{4}$, Hernandes Rufino dos Santos ${ }^{4}$

${ }^{1}$ Laboratório de Limnologia e Aquicultura (LLA), Curso de Biologia, Universidade Regional do Cariri (URCA), Crato-CE, Brasil.

${ }^{2}$ Programa de Pós-graduação em Agronomia (PPGA), Universidade Federal da Paraíba (UFPB), Areia-PB, Brasil.

${ }^{3}$ Setor de Vigilância Sanitária, VIGIÁGUA, Juazeiro do Norte-CE, Brasil.

${ }^{4}$ Laboratório de Tecnologia de Produtos (LTP), Curso de Agronomia, Universidade Federal do Cariri (UFCA), Crato-CE, Brasil.

*Correspondência: Programa de Pós-Graduação em Agronomia, Universidade Federal da Paraíba, Campus II, Rodovia PB-079, Areia, Paraíba, Brasil.CEP: 58.397-000.E-mail: iarley.toshik@gmail.com

\section{RESUMO}

A água é fundamental para a manutenção da vida, sendo a sua distribuição e controle de qualidade de indescritível importância para a população, no entanto, nem toda a população tem acesso à água de qualidade adequada. O presente trabalho teve como objetivo avaliar a adequação da Companhia de Água e Esgoto do Estado do Ceará (CAGECE) aos padrões de qualidade físico-químicas e microbiológicas da água de abastecimento público do município de Juazeiro do Norte, CE. Foram coletadas amostras nos anos de 2014 e 2015 pela CAGECE e o monitoramento foi realizado com 53 amostras mensais, divididas em duas coletas. Foram analisadas as variáveis: turbidez, cloro residual livre, coliformes totais e Escherichia coli. Os resultados do estudo apresentam uma situação preocupante com relação à qualidade da água distribuída pelo órgão supracitado, principalmente para os resultados das análises microbiológicas. Isso mostra a necessidade de intensificação no monitoramento e fiscalização nas condições da distribuição da água na cidade de Juazeiro do Norte, CE.

Palavras-chave: Monitoramento, Potabilidade, Distribuição.

\section{ABSTRACT}

Water is fundamental to life maintenance, being your distribution and quality control of great importance to the population, however, a part of the population do not have access to water with adequate quality. The present work has as objective evaluate the adequacy of the Water and Sewage Company of Ceará State (CAGECE) to the physicochemical and microbiological quality standards for public water supply in the municipality of Juazeiro do Norte, CE. Samples were collected in the years 2014 and 2015 by CAGECE and the monitoring was performed with 53 monthly samples, divided into two collections. It was done analyses of turbidity, free residual chlorine, total coliforms and Escherichia coli. The results of study show a worrisome situation about the quality of distributed water by the above-mentioned organ, mainly the results of microbiologic analysis. It shows the necessity of intensification in the monitoring and supervision of the conditions of water distribution in the city of Juazeiro do Norte, CE.

KeyWords: Monitoring, Potability, Distribution. 


\section{RESUMEN}

El agua es fundamental para el mantenimiento de la vida y su distribución y control de calidad de indecible importancia para la población, sin embargo, no toda la población tiene acceso a agua de calidad adecuada. El presente trabajo tuvo como objetivo evaluar la adecuación de la Empresa de Acueducto y Alcantarillado del Estado de Ceará los estándares de calidad físico-química y microbiológica del agua abastecimiento público del municipio de Juazeiro do Norte, CE. Las muestras se recolectaron en los años 2014 y 2015 por CAGECE y seguimiento se realizó con 53 muestras mensuales, divididas en dos colecciones. Se analizaron las variables: turbiedad, cloro residual libre, coliformes totales y Escherichia coli. Los resultados del estudio muestran una situación preocupante con respecto a la calidad del agua distribuida por la institución mencionada, principalmente por los resultados de las pruebas microbiológicas. Esto demuestra la necesidad de aumentar la vigilancia y supervisión de las condiciones de distribución de agua en la ciudad de Juazeiro do Norte, CE.

Descriptores: Monitoreo, Potabilidad, Distribución.

\section{INTRODUÇÃO}

A água é fundamental e insubstituível, sem ela não existe vida, pois todas as atividades do homem necessitam da mesma, sendo assim, cuidá-la é uma questão de sobrevivência (TELLES, 2013). De acordo com estatísticas do Projeto Brasil das Águas (2013) 70\% do planeta é constituído de água, sendo que somente $3 \%$ é de água doce e, desse total, $98 \%$ é subterrânea.

A falta de água, em quantidade ou em qualidade, é o principal empecilho ao desenvolvimento de regiões carentes e uma das principais razões de doenças ou morte prematura de pessoas (TELLES, 2013). Há de se refletir sobre qual água recebe-se através dos sistemas de abastecimento, as perdas provenientes do sistema, além de gerarem desperdício, também podem significar contaminação (CARVALHO et al., 2015).

A Companhia de Água e Esgoto do Estado do Ceará (CAGECE) é a empresa responsável pelo abastecimento humano da maior parte do estado do Ceará, atuando em 150 municípios, abastecendo mais de 5 milhões de habitantes. Na cidade de Juazeiro do Norte, contém um índice de atendimento de 84,93\% de ligações ativas. $\mathrm{O}$ abastecimento é garantido através dos mananciais compostos por quarenta e oito poços tubulares, os quais, antes de serem direcionados ao consumidor, são submetidos a um tratamento de forma a adequá-las ao padrão de potabilidade estabelecido pela Portaria 2914/11 do
Ministério da Saúde. O tratamento é realizado na Estação de Tratamento de Água (ETA), realizando processos de adição de produtos químicos, filtração e desinfecção com cloro (CAGECE, 2015).

A regulamentação do fornecimento da água deve seguir a Portaria No 2.914, de 12 de dezembro de 2011, do Ministério da Saúde, que dispõe sobre os procedimentos de controle e de vigilância da qualidade da água para consumo humano e seu padrão de potabilidade. Esta Portaria se aplica à água destinada ao consumo humano proveniente de sistema e solução alternativa de abastecimento de água.

À Secretaria de Vigilância em Saúde (SVS/MS) compete estabelecer diretrizes para a vigilância da qualidade da água para o consumo humano a serem implementadas pelos Estados, Distrito Federal e Municípios, respeitados os princípios do SUS e prioridades, objetivos, metas e indicadores do Programa Nacional da Vigilância da Qualidade da Água para Consumo Humano (VIGIAGUA). Este programa consiste no conjunto de ações adotadas continuamente para garantir que a água consumida pela população atenda ao padrão de potabilidade estabelecido na legislação vigente, bem como avaliar e prevenir os possíveis riscos que os sistemas e as soluções alternativas de abastecimento de água podem representar à população abastecida (BRASIL, 2011). 
O presente trabalho teve como objetivo avaliar a adequação da Companhia de Água e Esgoto do Estado do Ceará (CAGECE) aos padrões de qualidade físico-químicas e microbiológicas da água de abastecimento público do município de Juazeiro do Norte, CE.

\section{MATERIAIS E MÉTODOS}

O município de Juazeiro do Norte (Figura 1) está localizado a 377,3 metros de altitude, apresentando clima Tropical Quente Semiárido e Tropical Quente Semiárido Brando, com temperatura média entre 24 e $26{ }^{\circ} \mathrm{C}$. Seu Índice pluviométrico anual é em média 925,1 mm, com período chuvoso de janeiro a maio. Seu solo é predominantemente caracterizado por Aluviais e Latossolo Vermelho Amarelo (FUNCEME, 2015; IPECE, 2015). Com uma população estimada para o ano de 2016 de 268.248 habitantes (IBGE, 2016).

Figura 1. Localização geográfica do município de Juazeiro do Norte - CE.

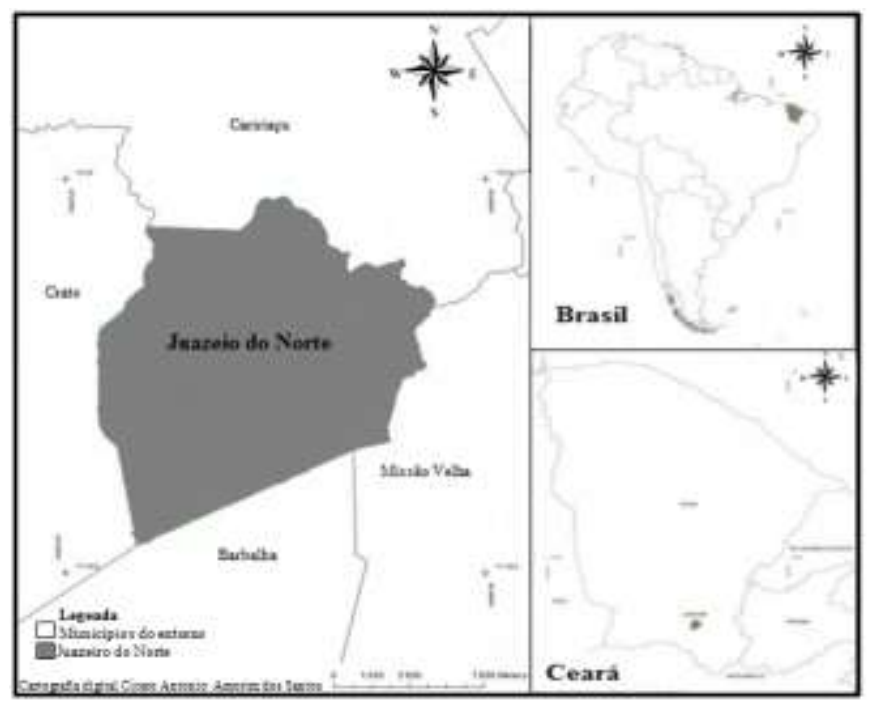

Fonte: IBGE (2011). Adaptado.

Atualmente, a vazão média distribuída na cidade de Juazeiro do Norte pela CAGECE é da ordem de 2.236,6 $\mathrm{m}^{3} \mathrm{~h}^{-1}$ de água potável, atendendo às exigências do Ministério da Saúde. Após o tratamento, a água é armazenada em 25 (vinte e cinco) reservatórios com capacidade total de 26.417 $\mathrm{m}^{3}$. A partir dessa etapa, a água é levada por gravidade para a distribuição através de adutoras ou tubulações de $\mathrm{PVC}, \mathrm{DEF}^{\circ} \mathrm{F}^{\mathrm{o}}$ e aço, com diâmetros que variam de 50 a $250 \mathrm{~mm}^{-1}$. A distribuição do sistema de abastecimento compreende uma malha de $985.488 \mathrm{~m}$ de rede, o que garante um índice de cobertura de 98,96\% do município. Os reservatórios em uso são limpos a cada seis meses e na rede de distribuição são executadas descargas periódicas para assegurar que a água distribuída não sofra alterações da qualidade (CAGECE, 2015).

Foram utilizados dados históricos das análises realizadas no ano de 2014 e 2015 pela Agência de Vigilância Ambiental da cidade de Juazeiro do Norte, que realiza o monitoramento da qualidade da água, distribuída pela CAGECE. O monitoramento foi realizado com 53 amostras mensais, divididas em duas coletas. As mesmas foram realizadas no início do mês com 27 amostras e outra no fim com 26 amostras, de forma aleatória em todo o município. Esse monitoramento foi feito onde ocorre o maior número de doenças de veiculação hídrica.

A coleta é realizada impreterivelmente na primeira torneira do local ao qual se deseja coletar, sendo necessário ter certeza que a água seja proveniente da rede de distribuição e não de caixas ou reservatórios internos. Deixa-se escoar por dois a três minutos, ou o tempo suficiente para eliminar a água estagnada na tubulação, havendo antes da coleta uma esterilização da torneira com aplicação de uma solução de hipoclorito de sódio (100mg L-1 ${ }^{1}$ ).

A coleta foi realizada em recipientes adequados, enviado pelo Ministério da Saúde, os quais foram identificados, posteriormente armazenados e encaminhados ao Laboratório Central de Saúde Pública (LACEN/CE). Nesse laboratório 
foram realizadas as análises de acordo com os requisitos especificados na NBR ISO/IEC 17025:2005. Os parâmetros previstos nesta Portaria devem atender às normas nacionais ou internacionais mais recentes, tais como: Standard Methods for the Examination of Water and Wastewater, de autoria das instituições American Public Health Association (APHA); American Water Works Association (AWWA) e Water Environment Federation (WEF); United States Environmental Protection Agency (USEPA); Normas publicadas pela International Standartization Organization (ISO) e Metodologias propostas pela Organização Mundial da Saúde (OMS), segundo Portaria 2.914/2011 (BRASIL, 2011).

As variáveis analisadas foram: turbidez, cloro residual livre, coliformes totais e E. coli. Após a liberação dos resultados das análises, a vigilância sanitária realizou a comparação dos resultados com os valores determinados na resolução da Portaria 2.914/11/MS, que possibilitou a classificação dos dados de forma sistemática possibilitando o fornecimento de respostas ao fenômeno pesquisado.

\section{RESULTADOS E DISCUSSÃO}

Na figura 2 estão representadas as análises de cloro residual livre e de turbidez da água de abastecimento público fora dos padrões no ano de 2014 e 2015 na cidade de Juazeiro do Norte.

Figura 2. Resultado do percentual de amostras de Cloro Residual Livre e Turbidez para consumo humano da água de abastecimento público fora dos padrões da cidade de Juazeiro do Norte-CE, no ano de 2014 (A) e 2015 (B).
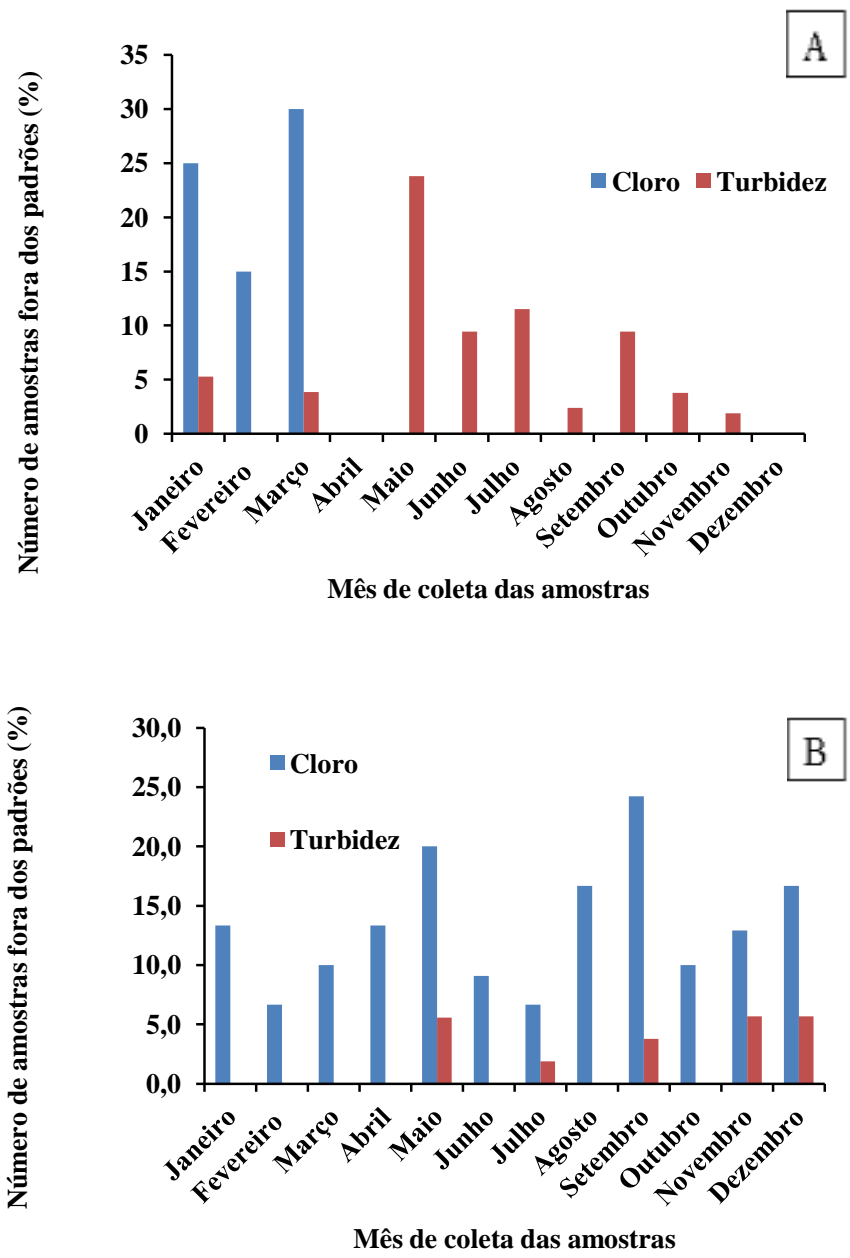

Fonte: VIGIAGUA (2015).

A variável cloro residual livre (CRL) no ano de 2014 (Figura 2A) foi analisada apenas nos meses de janeiro, fevereiro e março, sendo que os dados dos outros meses não constam no sistema de monitoramento. Para essa análise ocorreu uma variação de 15 a 30\% do percentual de amostras fora dos padrões, constatando o maior percentual no mês de março. A Turbidez, nesse mesmo ano, apresentou uma variação percentual de 1,9 a 23,8 \% de amostras fora dos padrões, sendo que no mês de novembro foi registrado o menor valor e o maior no mês de maio. No entanto, nos meses de fevereiro, abril e dezembro as amostras encontraram-se dentro da regulamentação.

No ano de 2015 (Figura 2B) o CRL apresentou resultados fora do recomendado em todas 
as coletas, variando de 6,7 a $24,2 \%$, sendo o maior percentual do número de amostra no mês de setembro.

Morais et al. (2016), ao avaliarem a quantidade de CRL da água de abastecimento três bairros da cidade de Rio Verde, GO, apenas um bairro se manteve no nível de referência recomendado pelo Ministério da Saúde em todas as coletas, os demais encontravam-se acima ou abaixo do recomendado durante o período de coleta. A mesma variação pode ser observada no presente trabalho, onde, no ano de 2015, todas as coletas apresentaram irregularidades.

De acordo com a Portaria 2.914/2011/MS (BRASIL, 2011) é obrigatória a manutenção de, no mínimo, 0,2 $\mathrm{mg} \mathrm{L}^{-1}$ de CRL em toda a extensão do sistema de distribuição. Valores abaixo do recomendado aumentam a probabilidade de presença de coliformes fecais e, por outro lado, altos valores podem também proporcionar outros problemas de saúde para a população (FREITAS et al., 2001).

A turbidez no ano de 2015 apresentou o percentual de amostra fora do recomendado de 1,9 a 5,7 , sendo que os maiores valores ocorreram em novembro. Porém, nos demais meses estudados, todas as amostras apresentaram-se dentro dos padrões recomendados.

O percentual de amostras com turbidez alta pode ser atribuído à intermitência do sistema, que possibilita a entrada de água contaminada no interior da tubulação vazia, onde a pressão é negativa. Isto pode indicar ser eventual ou pontual, uma vez que a rede de distribuição, em diversos locais encontra-se, com perfurações clandestinas (FREITAS et al., 2001).

De acordo com Portaria 2.914/2011/MS (BRASIL, 2011), o limite máximo para qualquer amostra pontual deve ser de 5,0 uT, assegurando, simultaneamente, o atendimento ao VMP de 5,0 uT em toda a extensão do sistema de distribuição.

A elevada taxa de turbidez na água forma flocos pesados que decantam mais rapidamente. Também acarreta maior dificuldade da desinfecção pela proteção que ele pode propiciar aos microrganismos (FUNASA, 2009).

Os resultados das análises biológicas de coliformes totais (CT) e de E. coli encontrados nas amostras analisadas estão representados na figura 3 .

Figura 3. Resultado percentual de amostras com presença de coliformes totais e E. coli em água de abastecimento público da cidade de Juazeiro do Norte, CE, no ano de 2014 (A) e 2015 (B).
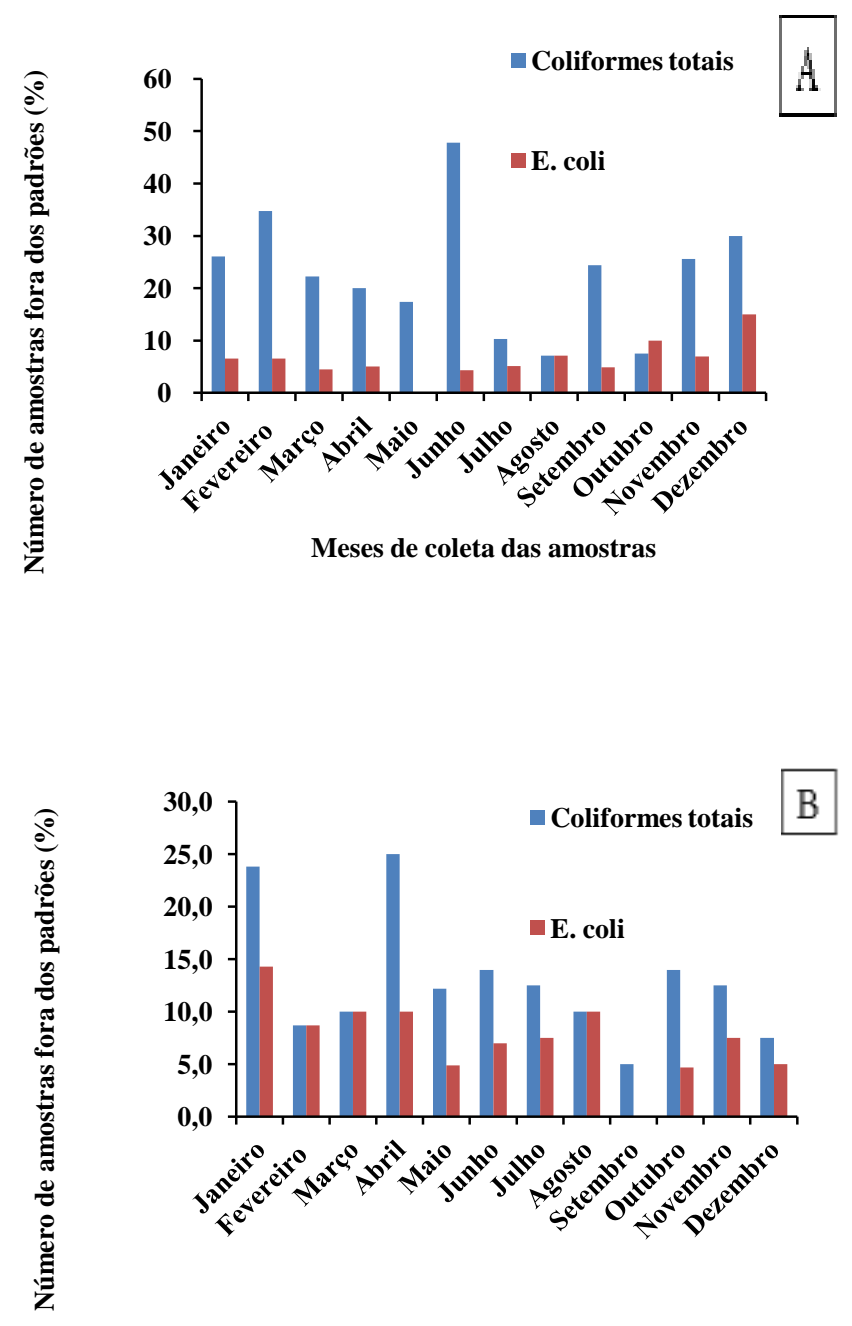

Meses de coleta das amostras

Fonte: VIGIAGUA (2015). 
Em todos os meses do ano de 2014 (Figura 3A) foi observada a presença de CT, com um percentual de 7 a $48 \%$ das amostras analisadas, sendo que a menor foi no mês de outubro e a maior porcentagem em junho. $\mathrm{O}$ mês de maio foi o único que não constatou presença de E. coli nas análises, sendo que o percentual de amostras que verificou presença foi de 4,3 a $15 \%$, com o menor valor no mês de junho e maior em dezembro.

No ano de 2015 (Figura 3B) também se constatou presença de CT em todas as coletas, com o percentual variando de 5 a $25 \%$ das amostras analisadas, com o menor valor em setembro e maior valor em abril. Observou-se, assim, que houve uma redução no ano de 2015 de amostras com presença de CT em relação ao ano de 2014. Setembro foi o único mês que não apresentou positividade para a presença de E. coli. O percentual de amostra com presença variou de 4,7 a $14,3 \%$, com o menor valor em outubro e o maior em janeiro. Constatando que os dois anos tiveram percentuais semelhantes de amostras com presença dessa bactéria.

Costa et al. (2012), avaliando a qualidade da água subterrânea de poços no estado do Ceará, constataram que $40 \%$ das amostras confirmaram a presença de CT e $12 \%$ de E. coli. Essas contaminações foram explicadas pela perfuração dos poços próximos a fossas sépticas ou falta de cuidados, já no presente trabalho, a justificativa seria pelo tratamento inadequado fornecido pela rede de abastecimento.

De acordo com Portaria 2.914/2011/MS (BRASIL, 2011) no sistema de distribuição (reservatórios e rede) deve haver sistemas ou soluções alternativas coletivas que abastecem a partir de 20.000 habitantes, com ausência em $100 \mathrm{~mL}^{-1}$ em 95\% das amostras examinadas no mês. Sabendo que a população estimada de Juazeiro do Norte é de
268.248 habitantes (IBGE, 2016), observa-se que os valores constatados neste trabalho para CT estão acima do permitido pela portaria que regulamenta a qualidade da água para consumo humano e seu padrão de potabilidade, chegando a obter presença de CT em até 48\% das amostras no ano de 2014.

Quando as propriedades da água não estão enquadradas aos limites de contaminação, a água se torna uma fonte de propagação de doenças, como a cólera, febre tifoide e várias outras (ROCHA, 2007).

A constatação de presença de CT não indica necessariamente que a água está contaminada por fezes e consequentemente por bactérias patogênicas, protozoários ou vírus, uma vez que sua presença pode ser detectada naturalmente na água, solo e plantas podendo ser organismos não essencialmente intestinais (RUOCCO, 2010).

De acordo com Portaria 2.914/2011/MS (BRASIL, 2011) que regulamenta o padrão microbiológico da água para consumo humano, a água deve apresentar ausência de $100 \%$ em todas as amostras. Sendo assim, as únicas amostras que se enquadraram neste parâmetro foram as dos meses de maio de 2014 e as de setembro de 2015. Os demais dados demonstraram que foi encontrado um percentual de $15 \%$ das amostras de água imprópria para o consumo humano.

A presença de E. coli ou de coliformes termotolerantes fornece evidência de contaminação fecal e sua detecção deve levar em consideração mais intervenção, que pode incluir mais amostragem e investigação de origens potenciais, tais como falhas na integridade do sistema de distribuição (SILVA, 2006; RASELLA, 2013). Outro fator pode ser o CRL abaixo do valor recomendado, que é de $0,2 \mathrm{mg} \mathrm{L}^{-1}$, o que pode justificar a presença de coliformes fecais na água da rede de abastecimento (FREITAS et al., 2001). 
O percentual médio de amostras de água satisfatórias para consumo humano no Estado da Bahia foi de $78 \%$, enquanto que $22 \%$ corresponderam as amostras de água insatisfatórias referentes aos ensaios físico-químicos e microbiológicos (BARRETO et al., 2015). Dados estes coletados na rede estadual de laboratórios de Vigilância da Qualidade da Água (BA). Sendo assim, verifica-se que este problema não é exclusivamente de Juazeiro do Norte, CE, revelando a necessidade de investimentos em ações articuladas entre os envolvidos com a qualidade da água, com vistas à efetividade na avaliação do risco que a água representa para a saúde coletiva.

No sistema de distribuição da água potável pode haver uma série de mudanças na sua composição (física, química ou biológica), tornando a qualidade da água na torneira diferente da que deixa a estação de tratamento, tais mudanças podem ser causadas por variadas perdas de integridade do sistema ou variações químicas (DEININGER et al., 1992).

No entanto, como pode ser visto nos relatórios anuais para informação ao consumidor publicado pela CAGECE, assegura-se a todos os consumidores o direito à informação sobre a qualidade da água potável, de acordo com os termos do Decreto 5440/05 do Código de Defesa do Consumidor Lei $\mathrm{n}^{\circ} 8.078$ e também atendendo a Portaria 2.914/2011/MS.

De acordo com o relatório, as medidas de monitoramento são realizadas semanalmente com amostras coletadas em pontos estratégicos (interesse sanitário). Realiza-se nas amostras avaliações laboratoriais de forma a assegurar a manutenção do padrão de potabilidade até a ligação do cliente. Quando ocorre algum resultado fora do padrão estabelecido (Portaria 2.914/2011/MS), as seguintes medidas são tomadas: 1- O laboratório de controle de qualidade comunica imediatamente o setor operacional desta operadora; 2- São efetuadas descargas de rede, isto é, por meio de registros existentes na rede de distribuição deixa-se correr a água para efetuar a limpeza da canalização; 3Verifica-se a ocorrência de alguma interferência próxima ao ponto onde foi coletada a amostra com resultado desfavorável; 4- Coleta-se nova amostra para verificar se os procedimentos surtiram efeito (CAGECE, 2015).

No relatório da CAGECE contém tabelas com os resultados das análises da água distribuída dos seguintes parâmetros: E. coli, CT, CRL, cor e turbidez. De acordo com a CAGECE no período de 01/01/2014 até 31/12/2014 foram coletadas 2425 amostras na rede de distribuição, das quais foram realizadas 9705 análises, e no período de 01/01/2015 até $31 / 12 / 2015,1675$ amostras na rede de distribuição, das quais foram realizadas 8437 análises de acordo com dados apresentados.

\section{CONCLUSÃO}

A partir das variáveis analisadas nesse trabalho, contatou-se que estas apresentaram-se fora dos padrões aceitáveis na maioria dos meses avaliados, tomando destaque as análises microbiológicas. Isso mostra a necessidade de intensificação no monitoramento e fiscalização nas condições da distribuição da água de abastecimento humano na cidade de Juazeiro do Norte, CE.

Todos os autores declararam não haver qualquer potencial conflito de interesses referente a este artigo.

\section{REFERÊNCIAS}

BARRETO, R.L.; PEDREIRA, M.M.; WILL, R.M. Monitoramento da qualidade da água para consumo humano no estado da Bahia no ano 2014. Revista Baiana de Saúde Pública, v.39, p.31-40, 2015. 
BRASIL. Ministério da Saúde. Secretaria de Vigilância em Saúde. Diretriz Nacional Do Plano de Amostragem da Vigilância da Qualidade da Água Para Consumo Humano. Diário Oficial da União, Brasília, DF, 2011.

CAGECE- Companhia de Água e Esgoto do Estado do Ceará. Relatório Anual Para Informação Ao Consumidor (2015). Disponível em: $<$ https://www.cagece.com.br/abastecimento-deagua/relatorios-de-qualidade/category/156-j-1 $>$.

Acesso em: 25 Out 2016.

CAGECE- Companhia de Água e Esgoto do Estado do Ceará. Relatório anual para informação ao consumidor (2014). Disponível em: $<$ https://www.cagece.com.br/abastecimento-deagua/relatorios-de-qualidade/category/164-j-1 $>$.

Acesso em: 25 Out 2016.

CARVALHO, W.S.; DOURADO, J.D.A.; FERNANDES, P.S.R.; BERNARDES, B.O.; MAGALHÃES, C.R.Consumo e perda de água potável na região metropolitana do Rio de Janeiro.

Revista Produção e Desenvolvimento, v.1, n.3, p.80-89, 2015.

COSTA, C. L.; LIMA, R. F.; PAIXÃO, G. C.; PAMTOJA, D. M. Avaliação da qualidade das águas subterrâneas em poços do estado do Ceará, Brasil. Semina: Ciências Biológicas e da Saúde, v.33, n.2, p.171-180, 2012.

DEININGER, R. A.; CLARK, R. M.; HESS, A. F.; BERNSTAM, E. V. Animação e visualização dos sistemas de distribuição da qualidade da água. Journal of the American Water Works Association. 1992.

FREITAS, M.B.; BRILHANTE, O.M.; ALMEIDA, L.M. Importância da análise de água para a saúde pública em duas regiões do Estado do Rio de Janeiro: enfoque para coliformes fecais, nitrato e alumínio. Cadernos de Saúde Pública, v.17, n.3, p.51-660, 2001.

FUNASA. Manual Prático de Analise de Água. $3^{\circ}$ edição. Brasília: Fundação Nacional de Saúde, 2009.

FUNCEME. Fundação Cearense de Meteorologia e Recursos Hídricos. 2015. Portal Hidrológico do Ceará. Disponível em: <http://www.hidro.ce.gov.br/>. Acesso em: 15 Out 2015.
IBGE. Instituto Brasileiro de geografia e Estatística. 2016.2 Disponível em:<http://cod.ibge.gov.br/7L5> Acesso em: 26 Out 2016.

IPECE. Instituto de Pesquisa e Estratégia Econômica do Ceará. 2015. Perfil básico municipal 2014 Cedro. Disponível em: <http://www.ipece.ce.gov.br/publicacoes/perfil_basic o/perfil-basico-municipal-2015.html > . Acesso em: 15 Fev 2015.

PORTARIA n 2.914, de 12 de dezembro de 2011. Dispõe sobre os procedimentos de controle e de vigilância da qualidade da água para consumo humano e seu padrão de potabilidade. Ministério da Saúde. Disponível em: http://bvsms.saude.gov.br/bvs/saudelegis/gm/2011/prt

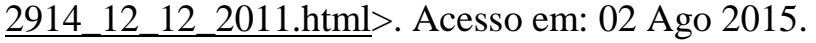

PROJETO BRASIL DAS ÁGUAS. 2013. Disponível em:

<http://brasildasaguas.com.br/educacional/aimportan cia-da-agua >. Acesso em: 27 mai 2014.

MORAIS, W. A.; SALEH, B. B.; ALVES, W. S.; AQUINO, D. S. Qualidade sanitária da água distribuída para abastecimento público em Rio Verde, Goiás, Brasil. Cadernos Saúde Coletiva, v.24, n.3, p.361-367, 2016.

RASELLA, D. Impacto do Programa Água para Todos (PAT) sobre a morbimortalidade por diarreia em crianças do estado da Bahia, Brasil. Cadernos de Saúde Pública, v.29, p. 40-45, 2013.

ROCHA, H. Quarenta Anos de História da Saneago. Goiânia: Poligráfica, 2007.

RUOCCO, B.P. análises microbiológicas de água tratada em creches municipais na região do São Francisco em Foz do Iguaçu-PR. Foz do Iguaçu, 2010.

SILVA, M.P; CAVALLI, D.R; OLIVEIRA, T.C.R.M. Avaliação do padrão coliformes à $45^{\circ} \mathrm{C}$ e comparação da eficiência das técnicas dos tubos múltiplos e petrifilm EC na detecção de coliformes totais e Escherichia coli em alimentos. Ciência e Tecnologia de Alimentos, v.26, n.2, p.352-359, 2006.

TELLES, D.D.A. Ciclo ambiental da água: da chuva à gestão. São Paulo: Editora Edgard Blucher, 2013. $504 \mathrm{p}$. 